\title{
A pplication of turmeric dye in the coating of Triphala guggle ayurvedic tablet
}

\author{
A njali Goel*, M.K. Bhardwaj ${ }^{1}$ and Neetu R ani \\ Department of Chemistry, KGM, IInd campus of Gurukula Kangri University, Haridwar (Uttarakhand), INDIA \\ 'Patanjali Ayurved Limited, Haridwar (Uttarakhand), INDIA \\ *Corresponding author. E-mail: anjaligoel10@gmail.com
}

\begin{abstract}
The aim of present work was to extract a yellow colour dye from turmeric rhizome (Curcuma longa) and to use it in the coating of triphala guggle ayurvedic (abbreviated as TGA) tablets. For coating of the TGA tablets the work was completed into two parts- in the first part extraction of dye from turmeric rhizome was carried out and in the second part extracted dye was used to coat the TGA-tablets. The dye extract was used in two forms for dyeing the tablets - firstly as liquid turmeric dye extract (LTDE) and secondly as powdered turmeric dye extract (PTDE). The optimum extraction conditions were settled after studying the effect of solvent, stirring time and temperature on the extraction of dye. The solvents used are water, ethanol and water- ethanol mixture. It was observed that the optimum extraction conditions for turmeric dye extraction were $60^{\circ} \mathrm{C}$ and 75 min stirring time with ethanol as solvent but from industrial point of view it is more economical to use ethanol-water (1:1) mixture for extraction. The extracted LTDE and PTDE were used to coat the TGA tablets. The results showed that the tablet coated with LTDE faded just in 30 days while PTDE coated tablets remained stable up to 40 days. Thus, present coating was suitable for coating the TGA tablets but the environmental factors like temperature and humidity influenced the stability of the coating a lot. The suitability of coating was studied by determining some physico- chemical parameters like average weight, diameter, thickness, friability, disintegration time and loss on drying of LTDE and PTDE coated tablets. The results were within the permissible limit of Indian Pharmacopea and other pharmacopeia. Therefore, coating of tablets with LTDE and PTDE both were found to be suitable for coating the TGA tablet.
\end{abstract}

Keywords : Triphala guggle, Natural dye, Turmeric rhizome, Extraction

\section{INTRODUCTION}

Importance of natural dyes is more relevant world-wide due to their non toxic, biodegradable and eco-friendly properties. Inspite of all, the present international consumption of natural dye is around $10,000 \mathrm{t}$, which is about $1 \%$ of the synthetic dye consumption. In many cases the fastness properties obtained by natural dyes are inferior to those obtained by synthetic dyes. The amount of research efforts devoted to natural dyes is negligible. It is all due to the existing limitations and technical drawbacks of these dyes, which restrict their use in pharma and dyeing industries (Siva, 2007; Singh and Singh, 2002; Gulrajni, 2001; Kapoor and Pushpgandhan, 2002).

Curcuma longa $\mathrm{L}$. is an important spice, used as a cosmetic and colouring agent, has also been used in indigenous system of medicine (Ramprashad and Sirse, 1956; Sachan and Kapoor, 2007). The origin of plant Curcuma longa L., which belongs to Zingiberaceae family is India. The plant is distributed throughout tropical and subtropical region of the world, being widely cultivated in south east Asian countries (Stankovic, 2004). Turmeric the brightest of naturally occurring yellow dye, a powerful antiseptic, which revitalizes the skin, is environment friendly (Mahanta and Tiwari, 2005). Turmeric is mainly valued for its principal colouring constituent curcumin, which imparts yellow colour on textile and food items (Khanna, 1999; Gulrajni et al., 1992). The $\lambda_{\text {max }}$ as determined spectroscopically was $426 \mathrm{~nm}$ for extracted turmeric dye as reported earlier and 420 for curcumin. (Singh, 1985; Hendry and Houghton, 1992). It is a fat soluble pigment but insoluble in aqueous medium. It can be made soluble in water by the use of diluent like propylene glycol and tween. Curcumin is sensitive to light but is moderately stable to heat. It also exhibits antioxidant property due to the presence of phenolic group (Showbagya et al., 2005). The maximum acceptable daily intake for curcumin is $0.1 \mathrm{mg} / \mathrm{kg}$ body weight (FAO, 2000).

In pharmaceutical industry tablets have been coated in many ways to enhance their market appeal and to enhance their swalloability. Untill about 1950, sugar was first choice as coating agent for pharmaceutical preprations and much time and efforts were spent in perfecting the sugar coating technique and nobody ever was concerned about the problems like material cost, toxic effect and pollution due to coating. In recent years there have been a number of developments in tablets coating technology. (Daher et al., 1999). Technology we consider almost 
routine today is really quite new to the pharmaceutical field. Colour, shape and size of final coated tablets are important for marketing strategies. Film coating protect the tablet from light, temperature and moisture, mask undesirable taste or odour, improves the appearance, provide tablet identity, facilitate swallowing and control or modify the release of the drug. Aqueous film coating is applied as a thin polymeric film to the surface of tablet. Solvent based coating of oral solid dosage has been rapidly replaced by aqueous coating for safety, environmental and economic reasons. However since tablet may contain moisture sensitive drug or excipient, the use of water raises concerns about the physical and chemical stability of the coated tablet (Cole, 1998 and Ruotsalainen, 2003). Film coating of tablet is a multivariate process, with many diffirent factors such as coating equipment, process conditions, composition of the core tablet and coating liquid, which affect the final product (Sheth et al., 2009). The aim of present work was to extract a yellow colour dye from turmeric rhizome (Curcuma longa) L. and to use it in the coating of herbal ayurvedic tablets of triphala guggle.

\section{MATERIALS AND METHODS}

The rhizome of Curcuma longa $L$. were collected from Haridwar market and identified by Quality Control Department, Patanjali Ayurved Limited, Haridwar (Indian Pharmacopia, 2007). All chemical and reagents used were of AR grade and double distilled water was used throughout the analysis.

For coating of the TGA tablets the work has been completed into two parts (1) Optimization of extraction conditions, (2). Coating of TGA tablets with dye extract and dye powder.

Extraction of turmeric dye - Rhizomes of Curcuma longa $\mathrm{L}$. were washed thoroughly with water and dried. These were then crushed in to coarse powder of particle size $0.50 \mathrm{~mm}$. Powdered raw material $(50 \mathrm{gm})$ was soaked in $(500 \mathrm{ml})$ of solvent (water / ethanol / 1:1 mixture of waterethanol) and kept over-night. The resulted coloured solution was subjected to heating with continuous stirring. The extracted solution was filtered and used in coating of tablets. Experiments were made to examine the technical feasibility of process at lab scale with reference to its yield and quality (Sachan et al., 2002).

In order to determine the optimum extraction conditions a number of experiments were performed with different solvents like water, ethanol, and water - ethanol mixture. 1. The first series of extraction was carried out with powdered turmeric rhizome soaked with distilled water in ratio of $1: 10$ at room temperature, $30^{\circ}$ and $60^{\circ} \mathrm{C}$ respectivly. At these temperature extraction was performed after 15, $30,45,60,75$ min stirring of the mixture.

2. In the second series of extraction of crushed turmeric rhizomes were soaked overnight in the solvent ethanol in the ratio of 1:10 and then subjected to stirring at room temperature, $30^{\circ}$, and $60^{\circ} \mathrm{C}$. The effect of different stirring time at $15,30,45,60,75 \mathrm{~min}$ was also studied.

3. In third series of extraction, the extraction was carried out with powdered turmeric rhizome with 1:1 mixture of ethanol-water in the ratio of 1:10 at room temperature, $30^{\circ}$, and $60^{\circ} \mathrm{c}$ and at different stirring time $15,30,45,60,75$ min respectively.

Coating of TGA tablets with LTDE and PTDE : For the coating of tablets, dye extract was used in two ways. In the first method dye extract was used as such for coating while in second method extract was converted in to powder form by crystallization at $-40^{\circ} \mathrm{C}$ (abbreviated as LTDE and PTDE respectively).

The coatings were applied on laboratory scale in a Pan Coating Apparatus (Hareson Pharma Pvt Ltd.). A high Shear Mixer (Remi Equipments Pvt.) is used to make the solution. Prepare $32 \%$ solution of Titanium dioxide in water, and $2.5 \%$ of Instacoat solution in isopropyl alcohol. Mix both the solution in a stainless-steel vessel with high shear mixer at a speed of 250-300 rpm for 20-30 min. Add $2.0-2.5 \%$ solution of turmeric dye powder with stirring till all dye get mixed properly. The tablets were film coated using a side vented perforated coating pan. Fixed quantity of tablets were kept in coating pan at bed temperature $45-50^{\circ} \mathrm{C}$. The various parameter like spray rate, inlet air temperature and rotating speed of pan were adjusted. After completion of coating tablets were dried at $60^{\circ} \mathrm{C}$ for $20 \mathrm{~min}$. Then tablets were removed from the pan and evaluated for physico-chemical parameters like average weight by Essae balance-Model DS-852J, diameter and thickness by Vernier calliper digital mitutoyo, friability by Friabilator Digital -TANCAO, hardness by Monsanto type tablet hardness tester and disintigration time by Harison pharma machinery Ltd.

Systronic -117, UV-vis spectrophotometer was used for the determination of $\lambda_{\max }$ of turmeric dye. The measurement of colour intensity of the extracted dye solution was made by Tintometer -F Lovibond. The colour intensity is considred as \% extraction of dye from rhizome.

\section{RESULTS AND DISCUSSION}

In order to determine the optimum extraction conditions sets of experiments were performed at different temperature, stirring time with different solvents. The results are presented in Figs. 1, 2 and 3 for the extraction of turmeric dye which show that the yield of the dye depends a lot on the extraction procedure and experimental conditions.

The results presented in Figs. 1, 2 and 3 for the extraction of turmeric dye show that the colour strength obtained with water, ethanol, and water-ethanol mixture follows the following order: Ethanol > ethanol-water mixture > 


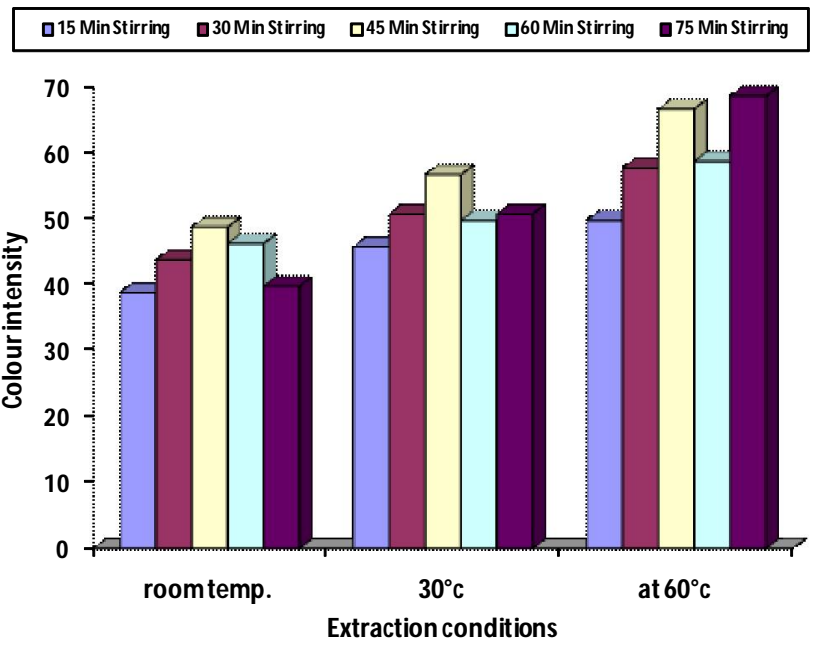

Fig. 1. Relative colour intensity with water.

water.

The colour strength with water ranges from 39-70 units while with ethanol and ethanol-water mixture, it is 43117and 41-99 units respectively (Sogi et al., 2010). The effect of temperature on the rate of extraction has been studied at room temperature, $30^{\circ}$, and $60^{\circ} \mathrm{c}$ respectively. The results presented in Figs. 1-3 clearly show that as the temperature increases the rate of extraction also increases. The colour strength of dye extracts obtained at room temperature is not good, it become slightly better at $30^{\circ} \mathrm{C}$ and maximizing when extractions are carried out at $60^{\circ} \mathrm{C}$. Extraction above $60^{\circ} \mathrm{C}$ with alcohol is not possible due low boiling point of alcohol. At $60^{\circ} \mathrm{C}$ the colour intensity increases from 49-69 with water, 82-119 with ethanol and 63-99 with ethanol-water mixture respectively (Umbreen et al., 2008). The colour intensity of the extracted dye has also been noted after different stirring time. It is also clear that as the time of stirring increases, the $\%$ of extracted dye also increases. In case of water it has been observed that initially from 15-45 min stirring, colour intensity increases but after more than $45 \mathrm{~min}$ stirring colour intensity decreases. But in ethanol and

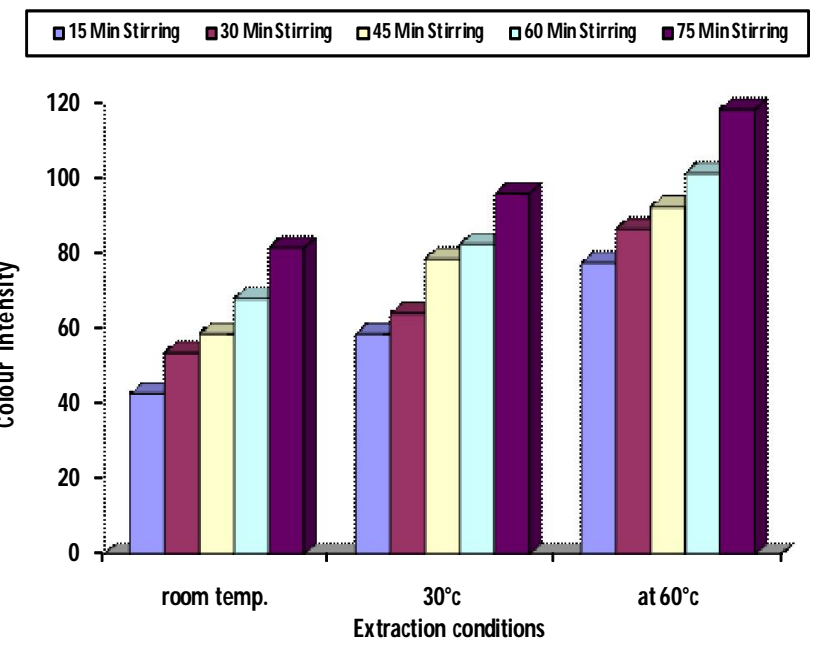

Fig. 2. Relative colour intensity with ethanol.

ethanol -water mixture, the rate of extraction increases with the increases of stirring time (Sogi et al., 2010).

Thus from the above results it can be concluded that the optimum extraction conditions for turmeric dye extraction are $60^{\circ} \mathrm{c}$ and $75 \mathrm{~min}$ stirring with ethanol as solvent. The results also show that maximum yield of dye was obtained with ethanol extraction but from industrial point of veiw it is more economical to use ethanol-water (1:1) mixture for extraction. Thus in the present study 1:1 water-ethanol mixture is used to extract the dye.

The TGA tablets coated with extracted dye are exposed to environmental conditions. The tablets were kept in atmospheric conditions during the month of June and August, 2010. The observations presented in Tables 1 and 2 show that between the room temperature in the range of $19-40^{\circ} \mathrm{C}$ and humidity conditions in the range of $0-65 \%$, the LTDE coated tablets are stable up to 30days, while PTDE coated tablets are stable up to 40 days after that fading starts. It is reported that the decomposition rate of turmeric dye at room temperature was faster than at refrigerator temperature. It may be due to the fast degradation of curcumin which is one of the main

Table 1. Results for the effect of temperature and humidity on the dye coated tablets.

\begin{tabular}{|c|c|c|c|c|c|}
\hline \multirow[t]{2}{*}{ S.N. } & \multirow{2}{*}{$\begin{array}{l}\text { Time } \\
\text { J une-A ugust, } 2010 \\
\text { Days }\end{array}$} & \multicolumn{2}{|c|}{ Tablet coated with LTDE } & \multicolumn{2}{|c|}{ Tablet coated with PTDE } \\
\hline & & $\begin{array}{l}\text { Room temp } \\
\left(19-40^{\circ} \mathrm{C}\right)\end{array}$ & $\begin{array}{c}\text { Humidity } \\
(65 \pm 5 \%)\end{array}$ & $\begin{array}{l}\text { Room temp } \\
\left(19-40^{\circ} \mathrm{C}\right)\end{array}$ & $\begin{array}{l}\text { Humidity } \\
(65 \pm 5 \%)\end{array}$ \\
\hline 1. & $0-10$ & Colour stable & Colour stable & Colour stable & Colour stable \\
\hline 2. & $10-20$ & Colour stable & Colour stable & Colour stable & Colour stable \\
\hline 3. & $20-30$ & Colour stable & Colour stable & Colour stable & Colour stable \\
\hline 4. & $30-40$ & Fading & Fading & Colour stable & Colour stable \\
\hline 5. & $40-50$ & Fading & Fading & Fading & Fading \\
\hline 6. & $50-60$ & Fading & Fading & Fading & Fading \\
\hline 7. & $60-70$ & Fading & Fading & Fading & Fading \\
\hline 8. & $70-80$ & Fading & Fading & Fading & Fading \\
\hline 9. & $80-90$ & Fading & Fading & Fading & Fading \\
\hline
\end{tabular}


Table 2. Results for coated tablets (before and after coating with turmeric dye).

\begin{tabular}{lcccc}
\hline Physico-chemical parameters & $\begin{array}{c}\text { Standard(I.P and In } \\
\text { house of Divya } \\
\text { Pharmacy) }\end{array}$ & Before coating & $\begin{array}{c}\text { After coating with } \\
\text { LTDE }\end{array}$ & $\begin{array}{c}\text { After coating with } \\
\text { PTDE }\end{array}$ \\
\hline 20 Tablet weight & $10.00 \pm 5 \%$ & $10.02 \mathrm{~g}$ & $10.17 \mathrm{~g}$ & $10.23 \mathrm{~g}$ \\
Average weight & $0.500+5 \%$ & $0.501 \mathrm{~g}$ & $0.508 \mathrm{~g}$ & $0.511 \mathrm{~g}$ \\
Average hardness & Not less then $1 \mathrm{~kg} / \mathrm{cm}^{2}$ & $1.51 \mathrm{~kg} / \mathrm{cm}^{2}$ & $2.12 \mathrm{~kg} / \mathrm{cm}^{2}$ & $2.43 \mathrm{~kg} / \mathrm{cm}^{2}$ \\
Average diameter & $10.40-10.80 \mathrm{~mm}$ & $10.41 \mathrm{~mm}$ & $10.67 \mathrm{~mm}$ & $10.78 \mathrm{~mm}$ \\
Average Thickness & $5.60-6.20 \mathrm{~mm}$ & $5.63 \mathrm{~mm}$ & $5.66 \mathrm{~mm}$ & $5.97 \mathrm{~mm}$ \\
Average Friability & Not more than $1 \%(\mathrm{I} . \mathrm{P})$ & $0.89 \% \mathrm{w} / \mathrm{w}$ & $0.39 \% \mathrm{w} / \mathrm{w}$ & $0.29 \% \mathrm{w} / \mathrm{w}$ \\
Disintegration time & Not more than $60 \mathrm{~min}(\mathrm{I} . P)$ & $38.56 \mathrm{~min}$ & $59.18 \mathrm{~min}$ & $59.19 \mathrm{~min}$ \\
Loss of drying(LOD) & Not more than $8 \% \mathrm{w} / \mathrm{w}$ & $7.61 \% \mathrm{w} / \mathrm{w}$ & $4.32 \% \mathrm{w} / \mathrm{w}$ & $5.96 \% \mathrm{w} / \mathrm{w}$ \\
\hline
\end{tabular}

I.P-Indian Pharmacopea

D15 Min stirring $\mathbf{Q} 30$ Min Stirring $\mathbf{D} 45$ Min stirring $\mathbf{D} 60$ Min stiring $\mathbf{Q} 75$ Min stiring

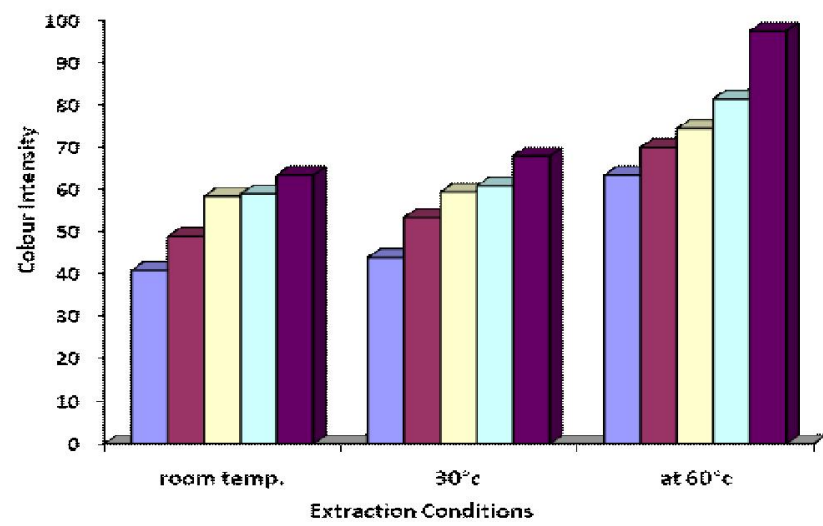

Fig. 3. Relative colour intensity with ethanol-water mixture.

constituent of turmeric dye (Tonnesen and Karlsen, 1985). Further the suitablity of the coating was checked by determining different parameters like average weight, diameter, thickness, LOD, disintegration time and friability (Table 2). The data show that the results of all parameters are within the prescribed limits. Average friability of coated tablets decreased from $0.89 \%$ to $0.39 \%$ and $0.29 \%$ while disintegration time increased from 38.56 to 59.18 and 59.19 mins. for LTDE and PTDE respectively. Initially loss on drying was $7.61 \%$, while after coating it has decreased to $4.32 \%$ and $5.96 \%$ respectively for LTDE and PTDE. The decrease in friability and loss on drying and increase in disintegration time are favourable for coated tablets. The coated tablets are useful to extend the shelf life of components and are sensitive to moisture or oxidation and to cover up the bed smell of the active ingredients (Anwar et al., 2007).

\section{Conclusion}

Thus, it was concluded that the coating with LTDE and PTDE are suitable for coating the TGA tablets but the environmental factors may influence the stability of the coating. It is recommended to protect the exposure of the coated tablets from environmental conditions.

\section{ACKNOWLEDGEMENTS}

Authors are thankful to Patanjali Ayurved Limited, Haridwar for providing practical support and to use their laboratories and instruments facilities.

\section{REFERENCES}

Anwar, E. Arsyadi, Broto, L.and Kardono, S. (2007). Study of coating tablet extract noni fruit (M orinda citrifolia,L.) with maltodextrin as a subcoating material. J. Med. Sci., 7(5) : 762-768.

Cole, G. C. (1998). Pharmaceutical Coating Technology, Taylor and Francis Ltd. 6-52.

Daher, L.J., Hayward, R.L. and Sackman, L.C. (1999). U.S Patent 5874108, 23 February (1999), Salt coating.

FAO (2000). Evaluation of certain food additives. FAO/WHO Expert committee report on food additives, WHO Technical Report Series 891, WHO Geneva, 26-27.

Gulrajani, M. L. (2001). Present status of natural dyes. Indian J. Fibre Text. Res., 26: 191-201.

Gulrajni, M.L, Gupta, D., Agarwal, V. and Jain, M. (1992) Some studies on natural yellow Dyes: Part I - CI Natural yellow 3: Turmeric. Indian Textile J ournal, Jan, 50-56.

Hendry, G.A.F. and Houghton, J.D. (1992). Natural Food Colourents, (Blackie and Son Ltd.).

Indian Pharmacopia (2007). 7: 2041-2047.

Kapoor,V.P. and Pushpgandhan, P. (2002). Natural dye based herbal Gulal, Natural Product Radiance, 1(2): 8-14.

Khanna, N.M. (1999). Turmeric-Nature's Precious gift. Current science, 76(10):1351-1356.

Mahanta, D. and Tiwari. S.C. (2005). Natural dye yielding plants and indigenous knowledge on dye prepration in Arunachal Pradesh, Northeast India, Cur rent science, (88): 1474-1480.

Ramprashad, C. and Sirse, M. (1956). Studies on Indian medicinal plants, Curcuma Ionga Linn- effect of curcumin and the essential oils of $C$. Ionga on bile secretion. J. Sci Ind Res, 15C, 262-265.

Ruotsalainen, M. (2003). Studies on aqueous film coating of tablets in side vented perforated pan coater. ISBN 352-101041-X Helsinki. 
Siva, R. (2007). Status of natural dyes and dye yielding plants in India, Current Science, 92 (7) : 916-925.

Sachan, K., Verma, C.I. and Kapoor, V.P. (2002). Variability in colour contest of Curcuma longa L. growing in different habitats, National conference biodiversity-Past \& Present, Birbal Sahani Institute of Palaeobotany, Lucknow, Nov, 28-29.

Sachan, K. and Kapoor, V.P. (2007). Optimization of extraction and dyeing conditions for traditional turmeric dye. National Botanical Research Institute, Lucknow, Indian. J ournal of Traditional Knowledge, 6 (2): 270-278.

Singh, V. and Singh, R.V. (2002). Healthy Hues, D own to E arth, (11) : 25-31.

Stankovic, I. (2004). FAO, Curcumin, Chemical and Technical Assessment (CTA), $61^{\text {st }}$ Joint expert committee on food additives, 1(8):1-8.

Showbagya, H.B. Samhita, S. Krishnamurty, S.R. and Bhattacharya, N. S. (2005). Stability of water soluble turmeric colorant in an extruded food product during storage.
J ournal of food Engineering, 67(3): 367-371.

Singh, A. (1985). Enhancement of turmeric (Curcuma longa) growth, rhizome yield and its yellow pigment content by processing chilling treatment to rhizomes. J India B ot. SoC, (64): 210-213.

Sogi, D.S. Sharma, S. Oberoi, P.S. and Wani, I.A. (2010). Effect of extraction parameters on curcumin yield from turmeric. J. Food Sci. Technol., 47(3) : 300-304.

Sheth Navin, Shah Sunny, Potdar Arti and Shah, Anand (2009). Studies in optimization of aqueous film coating parameters. International Journal of Pharmaceutical Science and Nanotechnology, 2 (3): 621-626.

Tonnesen, H.H. and Karlsen, J. (1985). Studies of curcumin and curcuminoids: VI. Kinetics of curcumin degradation in aqueous solutions. Z. Lebensm.Unters. Forsch,180: 402404

Umbreen, Saima Ali, Shaukat Hussain, Tanveer and Nawaz, Rakhshanda (2008). Dyeing properties of natural dyes extracted fron turmeric and their comparison with reactive dyeing. RJ TA, 12(4) : 1-11. 